Acta Crystallographica Section E

Structure Reports

Online

ISSN 1600-5368

\section{Andrei S. Batsanov ${ }^{a *}$ and Igor F. Perepichkab}

${ }^{a}$ Department of Chemistry, University of Durham, South Road, Durham DH1 3LE, England, and ${ }^{\mathbf{b}} \mathrm{L}$. M. Litvinenko Institute of Physical Organic and Coal Chemistry, National Academy of Sciences of Ukraine, Donetsk 83114, Ukraine

Correspondence e-mail:

a.s.batsanov@durham.ac.uk

\section{Key indicators}

Single-crystal X-ray study

$T=110 \mathrm{~K}$

Mean $\sigma(\mathrm{C}-\mathrm{C})=0.007 \AA$

$R$ factor $=0.067$

$w R$ factor $=0.172$

Data-to-parameter ratio $=10.5$

For details of how these key indicators were automatically derived from the article, see http://journals.iucr.org/e.

\title{
Ethyl 9-dicyanomethylene-2,5,7-trinitro- fluorene-4-carboxylate
}

The title compound, $\mathrm{C}_{19} \mathrm{H}_{9} \mathrm{~N}_{5} \mathrm{O}_{8}$, has a warped fluorene ring system due to steric repulsion between the 4-ethoxycarbonyl and 5-nitro groups.

\section{Comment}

The title compound, (I), has been obtained in the course of our studies of electron acceptors of the fluorene series and their charge-transfer complexes and radical ion salts with $\pi$ electron donors, e.g. tetrathiafulvalene family donors (Perepichka et al., 1998, 2000, 2002; Batsanov et al., 2001, 2002; Kuz'mina et al., 2002).

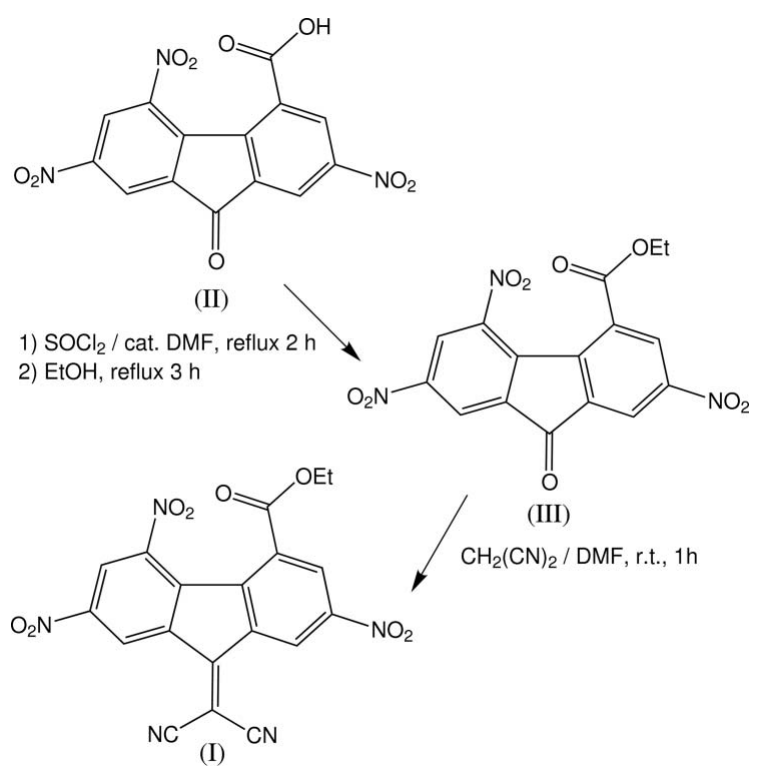

The molecular structure of (I) is strongly influenced by steric repulsion between the nitro and ethoxycarbonyl groups in positions 4 and 5, respectively. This overcrowding, indicated, for example, by the short (intramolecular) non-bonding

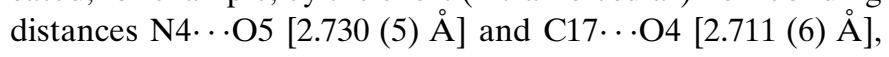
causes the above-mentioned substituents to tilt out of the fluorene plane in opposite directions. Furthermore, the fluorene aromatic system itself loses planarity and adopts a warped (twisted) conformation, the deviations $(\AA)$ of its $\mathrm{C}$ atoms from the mean plane being: C1 -0.186(4), C2 -0.149 (4), C3 0.135 (4), C4 0.245 (4), C5 -0.226 (4), C6 -0.145 (4), C7 $0.070(4)$, C8 $0.138(4)$, C9 0.033 (4), C10 -0.039 (4), C11 0.060 (5), C12 -0.013 (5) and C13 0.078 (4). Similar distortions are typical for other fluorene derivatives with bulky substituents in positions 4 and 5, e.g. 9-dicyanomethylene-2,4,5,7-tetranitrofluorene (Silverman et al., 1974;
Received 24 January 2006 Accepted 26 January 2006 


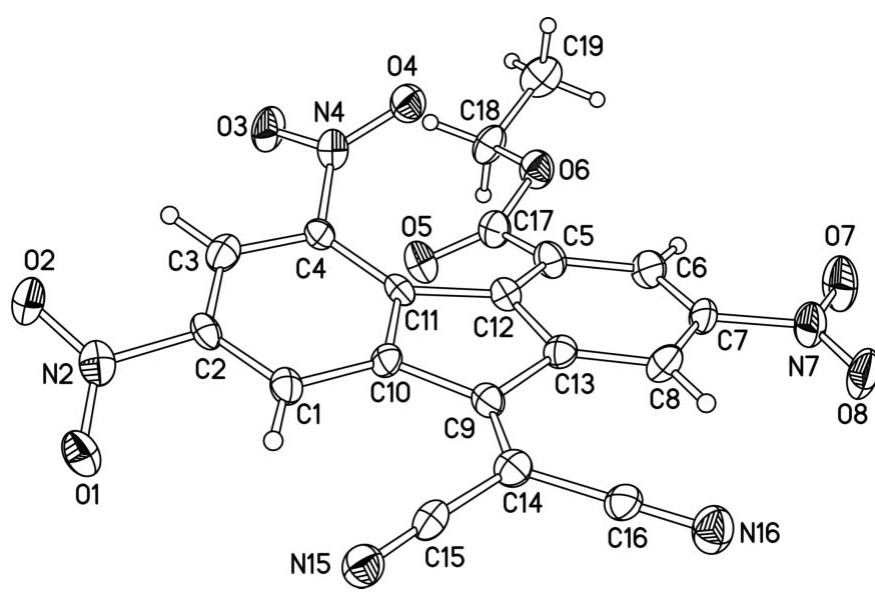

Figure 1

The molecular structure of (I). Atomic displacement ellipsoids are drawn at the $50 \%$ probability level.

Batsanov et al., 2001), 9-dicyanomethylene-4,5-dinitrofluorene-2,7-disulfonamide (Batsanov \& Perepichka, 2004) or 9-dicyanomethylene-4-bromo-2,5,7-trinitrofluorene (Perepichka et al., 2002).

\section{Experimental}

To a suspension of 2,5,7-trinitro-9-oxofluorene-4-carboxylic acid $(10.0 \mathrm{~g}, 27.8 \mathrm{mmol})$, (II), in thionyl chloride $(50 \mathrm{ml})$ a catalytic amount of $\mathrm{N}, \mathrm{N}$-dimethylformamide (2 drops) was added. The mixture was refluxed for $2 \mathrm{~h}$ (full dissolution occurring in 15-20 min) and then evaporated under reduced pressure until dry. Ethanol $(50 \mathrm{ml})$ was added to the residue, the mixture was refluxed for $3 \mathrm{~h}$ and left to cool for crystallization. The precipitate was filtered off, washed with cold ethanol $(2 \times 20 \mathrm{ml})$ and dried to yield crude compound (III) $(9.1 \mathrm{~g}, 84 \%$; m.p. 426-430 K). This was dissolved in boiling dioxane $(25 \mathrm{ml})$, filtered hot and the filtrate was diluted with hot ethanol $(100 \mathrm{ml})$. On cooling, the solid was filtered off, washed with ethanol and dried. This procedure was repeated to give pure compound (III) (7.2 g, 67\%) as yellow crystals (m.p. 434-435 K). ${ }^{1} \mathrm{H}$ NMR $\left(200 \mathrm{MHz}\right.$, acetone- $\left.\mathrm{d}_{6}\right): \delta 8.99(1 \mathrm{H}, d, J=2.0 \mathrm{~Hz}, \mathrm{H}-3), 8.83$ $(1 \mathrm{H}, d, J=2.0 \mathrm{~Hz}, \mathrm{H}-6), 8.78(1 \mathrm{H}, d, J=2.2 \mathrm{~Hz}, \mathrm{H}-8), 8.69(1 \mathrm{H}, d, J=$ $2.2 \mathrm{~Hz}, \mathrm{H}-1), 4.42(2 \mathrm{H}, q, J=7.2 \mathrm{~Hz}, \mathrm{CH} 2), 1.43(3 \mathrm{H}, t, J=7.2 \mathrm{~Hz}$, $\mathrm{CH} 3) .{ }^{13} \mathrm{C}$ NMR $\left(50 \mathrm{MHz}\right.$, acetone- $\left.d_{6}\right): \delta 186.21(\mathrm{C}=\mathrm{O}), 165.44(-$ $\left.\mathrm{CO}_{2}-\right), 150.71,150.56,147.36,144.19,140.30,140.08,139.18,132.90$, 131.05, 126.38, 122.95, 122.00, $63.84\left(\mathrm{CH}_{2}\right), 14.32\left(\mathrm{CH}_{3}\right)$. MS (EI): $\mathrm{m} / z$ $387\left(M^{+}, 100 \%\right)$. HRMS (EI): $\mathrm{m} / z$ 387.03447; calculated exact mass: 387.03388. Analysis found: C 49.52, H 2.40, N 10.89\%; $\mathrm{C}_{16} \mathrm{H}_{9} \mathrm{~N}_{3} \mathrm{O}_{9}$ requires: C 49.62, H 2.34, N 10.85\%.

Compound (III) $(5.0 \mathrm{~g}, 12.9 \mathrm{mmol})$ was dissolved in $\mathrm{N}, \mathrm{N}$ dimethylformamide $(25 \mathrm{ml})$, malonitrile $(2.2 \mathrm{~g}, 33.3 \mathrm{mmol})$ was added to this solution and the mixture was stirred at room temperature for $1 \mathrm{~h}$ (the product began to precipitate in $30 \mathrm{~min})$. 2-Propanol $(100 \mathrm{ml})$ was added to the mixture and it was allowed to stand at $273 \mathrm{~K}$ for 1$2 \mathrm{~h}$. The solid was filtered off, washed with 2-propanol and dried to yield crude compound (I) (5.2 g, 93\%; m.p. 539-543 K). It was dissolved in boiling dioxane $(75 \mathrm{ml})$, hot 2-propanol was added to the solution and the product left to crystallize. The solid was filtered off, washed with 2-propanol and dried. The purification procedure was repeated once more, to afford pure compound (I) $(4.8 \mathrm{~g}, 85 \%)$ as bright-yellow crystals (m.p. 543-545 K). ${ }^{1} \mathrm{H}$ NMR $(200 \mathrm{MHz}$, acetone- $d_{6}+$ half a drop $\left.\mathrm{CF}_{3} \mathrm{CO}_{2} \mathrm{D}\right): \delta 9.69(1 \mathrm{H}, d, J=2.0 \mathrm{~Hz}, \mathrm{H}-8)$,
$9.60(1 \mathrm{H}, d, J=2.0 \mathrm{~Hz}, \mathrm{H}-1), 9.04(1 \mathrm{H}, d, J=2.0 \mathrm{~Hz}, \mathrm{H}-6), 8.88(1 \mathrm{H}, d$ $J=2.0 \mathrm{~Hz}, \mathrm{H}-3), 4.43(2 \mathrm{H}, q, J=7.2 \mathrm{~Hz}, \mathrm{CH} 2), 1.44(3 \mathrm{H}, t, J=7.2 \mathrm{~Hz}$, $\mathrm{CH} 3) .{ }^{13} \mathrm{C}$ NMR $\left(100 \mathrm{MHz}\right.$, acetone- $d_{6}+0.5$ drop $\left.\mathrm{CF}_{3} \mathrm{CO}_{2} \mathrm{D}\right): \delta$ $165.30\left(-\mathrm{CO}_{2}-\right), 154.81,141.14,140.14,139.25,137.49,133.05,130.49$, 125.76, 124.75, 123.70, 121.52, 117.74, 113.96, 113.37, 113.32, 110.17, $63.96\left(\mathrm{CH}_{2}\right), 14.36\left(\mathrm{CH}_{3}\right)$. MS (EI): $m / z 435\left(M^{+}, 100 \%\right)$. Analysis found: $\mathrm{C} 52.52, \mathrm{H} 2.03, \mathrm{~N} 16.15 \% ; \mathrm{C}_{19} \mathrm{H}_{9} \mathrm{~N}_{5} \mathrm{O}_{8}$ requires: $\mathrm{C} 52.42, \mathrm{H}$ $2.08, \mathrm{~N} 16.09 \%$. Compound (I) was dissolved in hot acetonitrile and left to cool slowly to yield single crystals of X-ray quality.

\section{Crystal data \\ $\mathrm{C}_{19} \mathrm{H}_{9} \mathrm{~N}_{5} \mathrm{O}_{8}$ \\ $M_{r}=435.31$ \\ Monoclinic, $P 2_{1} / c$ \\ $a=19.481(2) \AA$ \\ $b=8.620$ (1) $\AA$ \\ $c=10.814(1) \AA$ \\ $\beta=99.40(1)^{\circ}$ \\ $V=1791.6(3) \AA^{3}$ \\ $Z=4$}

\section{Data collection}

Bruker SMART $1 \mathrm{~K}$ CCD area-

detector diffractometer

$\omega$ scans

Absorption correction: none

7993 measured reflections

3079 independent reflections

\section{Refinement}

Refinement on $F^{2}$

$R\left[F^{2}>2 \sigma\left(F^{2}\right)\right]=0.067$

$w R\left(F^{2}\right)=0.172$

$S=1.03$

3079 reflections

292 parameters

$\mathrm{H}$-atom parameters constrained

\author{
$D_{x}=1.615 \mathrm{Mg} \mathrm{m}^{-3}$ \\ Mo $K \alpha$ radiation \\ Cell parameters from 1107 \\ reflections \\ $\theta=3.1-22.6^{\circ}$ \\ $\mu=0.13 \mathrm{~mm}^{-1}$ \\ $T=110(2) \mathrm{K}$ \\ Prism, yellow \\ $0.18 \times 0.07 \times 0.07 \mathrm{~mm}$
}

$$
\begin{aligned}
& 1518 \text { reflections with } I>2 \sigma(I) \\
& R_{\text {int }}=0.126 \\
& \theta_{\max }=25.0^{\circ} \\
& h=-13 \rightarrow 22 \\
& k=-9 \rightarrow 10 \\
& l=-12 \rightarrow 12
\end{aligned}
$$

Table 1

Selected geometric parameters $\left(\AA{ }^{\circ}\right)$.

\begin{tabular}{llll}
\hline N15-C15 & $1.152(6)$ & C10-C11 & $1.412(6)$ \\
N16-C16 & $1.147(6)$ & C11-C12 & $1.502(7)$ \\
C9-C14 & $1.352(7)$ & C12-C13 & $1.397(7)$ \\
C9-C10 & $1.465(7)$ & C14-C16 & $1.444(7)$ \\
C9-C13 & $1.495(6)$ & C14-C15 & $1.446(7)$ \\
& & & \\
C2-C1-C10 & $116.9(4)$ & C6-C5-C12 & $117.7(5)$ \\
C3-C2-C1 & $123.3(4)$ & C7-C6-C5 & $119.2(5)$ \\
C2-C3-C4 & $118.0(5)$ & C6-C7-C8 & $124.3(5)$ \\
C3-C4-C11 & $121.4(4)$ & C7-C8-C13 & $116.0(5)$ \\
\hline
\end{tabular}

The diffraction was rather weak, with a mean $I / \sigma(I)$ ratio of 5.2. The methyl group was refined as a rigid body $(\mathrm{C}-\mathrm{H}=0.98 \AA)$ rotating around the $\mathrm{C}-\mathrm{C}$ bond, with a common (refined) $U_{\text {iso }}$ value for all three $\mathrm{H}$ atoms. Other $\mathrm{H}$ atoms were treated as riding in idealized positions, with $\mathrm{Cs} p^{3}-\mathrm{H}=0.99 \AA$ and $\mathrm{Cs} p^{2}-\mathrm{H}=0.95 \AA$, and $U_{\text {iso }}(\mathrm{H})=1.3 U_{\text {eq }}(\mathrm{C})$ and $1.2 U_{\text {eq }}(\mathrm{C})$, respectively.

Data collection: SMART (Bruker, 1997); cell refinement: SAINT (Bruker, 1999); data reduction: $S A I N T$; $\operatorname{program}(\mathrm{s})$ used to solve structure: SHELXTL (Bruker, 1997); program(s) used to refine structure: SHELXTL; molecular graphics: SHELXTL; software used to prepare material for publication: SHELXTL. 
The authors thank Professor M. R. Bryce for fruitful advice.

\section{References}

Batsanov, A. S., Bryce, M. R., Lyubchik, S. B. \& Perepichka, I. F. (2002). Acta Cryst. E58, o1106-o1110.

Batsanov, A. S. \& Perepichka, I. F. (2004). Acta Cryst. E60, o1892-o1894.

Batsanov, A. S., Perepichka, I. F., Bryce, M. R. \& Howard, J. A. K. (2001). Acta Cryst. C57, 1299-1302.

Bruker (1997). SMART (Version 5.054) and SHELXTL (Version 5.10). Bruker AXS Inc., Madison, Wisconsin, USA.

Bruker (1999). SAINT. Version 6.01. Bruker AXS Inc., Madison, Wisconsin, USA.
Kuz'mina, L. G., Perepichka, I. F., Perepichka, D. F., Howard, J. A. K. \& Bryce, M. R. (2002). Crystallogr. Rep. 47, 251-261.

Perepichka, D. F., Bryce, M. R., Perepichka, I. F., Lyubchik, S. B., Christensen, C. A., Godbert, N., Batsanov, A. S., Levillain, E., McInnes, E. J. L. \& Zhao, J. P. (2002). J. Am. Chem. Soc. 124, 14227-14238.

Perepichka, I. F., Kuz'mina, L. G., Perepichka, D. F., Bryce, M. R., Goldenberg, L. M., Popov, A. F. \& Howard, J. A. K. (1998). J. Org. Chem. 63, 64846493.

Perepichka, I. F., Popov, A. F., Orekhova, T. V., Bryce, M. R., Andrievskii, A. M., Batsanov, A. S., Howard, J. A. K. \& Sokolov, N. I. (2000). J. Org. Chem. 65, 3053-3063.

Silverman, J., Yannoni, N. F. \& Krukonis, A. P. (1974). Acta Cryst. B30, 14741480. 


\section{supporting information}

Acta Cryst. (2006). E62, o885-o887 [https://doi.org/10.1107/S1600536806003175]

\section{Ethyl 9-dicyanomethylene-2,5,7-trinitrofluorene-4-carboxylate}

\section{Andrei S. Batsanov and Igor F. Perepichka}

Ethyl 9-dicyanomethylene-2,5,7-trinitrofluorene-4-carboxylate

Crystal data

$\mathrm{C}_{19} \mathrm{H}_{9} \mathrm{~N}_{5} \mathrm{O}_{8}$

$M_{r}=435.31$

Monoclinic, $P 2_{1} / c$

Hall symbol: -P 2ybc

$a=19.481(2) \AA$

$b=8.620(1) \AA$

$c=10.814(1) \AA$

$\beta=99.40(1)^{\circ}$

$V=1791.6(3) \AA^{3}$

$Z=4$

\section{Data collection}

Bruker SMART 1 K CCD area-detector diffractometer

Radiation source: fine-focus sealed tube

Graphite monochromator

Detector resolution: 8 pixels $\mathrm{mm}^{-1}$

$\omega$ scans

7993 measured reflections

\section{Refinement}

Refinement on $F^{2}$

Least-squares matrix: full

$R\left[F^{2}>2 \sigma\left(F^{2}\right)\right]=0.067$

$w R\left(F^{2}\right)=0.172$

$S=1.03$

3079 reflections

292 parameters

0 restraints

Primary atom site location: structure-invariant direct methods

Secondary atom site location: difference Fourier map

Special details

Experimental. The data collection nominally covered over a hemisphere of reciprocal space, by a combination of 4 sets of $\omega$ scans; each set at different $\varphi$ and/or $2 \theta$ angles and each scan (46.5 sec exposure) covering $0.3^{\circ}$ in $\omega$. Crystal to detector distance $6.03 \mathrm{~cm}$.
$F(000)=888$

$D_{\mathrm{x}}=1.615 \mathrm{Mg} \mathrm{m}^{-3}$

Mo $K \alpha$ radiation, $\lambda=0.71073 \AA$

Cell parameters from 1107 reflections

$\theta=3.1-22.6^{\circ}$

$\mu=0.13 \mathrm{~mm}^{-1}$

$T=110 \mathrm{~K}$

Prism, yellow

$0.18 \times 0.07 \times 0.07 \mathrm{~mm}$

3079 independent reflections 1518 reflections with $I>2 \sigma(I)$

$R_{\text {int }}=0.126$

$\theta_{\max }=25.0^{\circ}, \theta_{\min }=2.1^{\circ}$

$h=-13 \rightarrow 22$

$k=-9 \rightarrow 10$

$l=-12 \rightarrow 12$

Hydrogen site location: inferred from neighbouring sites

$\mathrm{H}$-atom parameters constrained

$w=1 /\left[\sigma^{2}\left(F_{\mathrm{o}}^{2}\right)+(0.0632 P)^{2}+0.5406 P\right]$

where $P=\left(F_{\mathrm{o}}{ }^{2}+2 F_{\mathrm{c}}{ }^{2}\right) / 3$

$(\Delta / \sigma)_{\max }<0.001$

$\Delta \rho_{\max }=0.41 \mathrm{e} \AA^{-3}$

$\Delta \rho_{\min }=-0.37$ e $\AA^{-3}$

Extinction correction: SHELXTL, $\mathrm{Fc}^{*}=\mathrm{kFc}\left[1+0.001 \times \mathrm{Fc}^{2} \lambda^{3} / \sin (2 \theta)\right]^{-1 / 4}$

Extinction coefficient: 0.018 (2) 
Geometry. All e.s.d.'s (except the e.s.d. in the dihedral angle between two 1.s. planes) are estimated using the full covariance matrix. The cell e.s.d.'s are taken into account individually in the estimation of e.s.d.'s in distances, angles and torsion angles; correlations between e.s.d.'s in cell parameters are only used when they are defined by crystal symmetry. An approximate (isotropic) treatment of cell e.s.d.'s is used for estimating e.s.d.'s involving 1.s. planes.

Refinement. Refinement of $F^{2}$ against ALL reflections. The weighted $R$-factor $w R$ and goodness of fit $S$ are based on $F^{2}$, conventional $R$-factors $R$ are based on $F$, with $F$ set to zero for negative $F^{2}$. The threshold expression of $F^{2}>\sigma\left(F^{2}\right)$ is used only for calculating $R$-factors(gt) etc. and is not relevant to the choice of reflections for refinement.

Fractional atomic coordinates and isotropic or equivalent isotropic displacement parameters $\left(\AA^{2}\right)$

\begin{tabular}{|c|c|c|c|c|}
\hline & $x$ & $y$ & $z$ & $U_{\text {iso }} * / U_{\text {eq }}$ \\
\hline $\mathrm{O} 1$ & $0.53613(17)$ & $0.2322(4)$ & $0.2237(4)$ & $0.0309(9)$ \\
\hline $\mathrm{O} 2$ & $0.55693(17)$ & $0.2996(4)$ & $0.4205(3)$ & $0.0321(10)$ \\
\hline $\mathrm{O} 3$ & $0.34804(18)$ & $0.4135(4)$ & $0.6081(3)$ & $0.0330(10)$ \\
\hline $\mathrm{O} 4$ & $0.28475(17)$ & 0.5939 (4) & $0.5031(3)$ & $0.0293(9)$ \\
\hline O5 & $0.21531(18)$ & $0.2915(4)$ & $0.4372(3)$ & $0.0323(10)$ \\
\hline O6 & $0.13270(17)$ & $0.4589(4)$ & $0.4749(3)$ & $0.0302(10)$ \\
\hline $\mathrm{O} 7$ & $-0.00294(18)$ & $0.6632(5)$ & $0.0855(4)$ & 0.0407 (11) \\
\hline O8 & $0.04476(17)$ & $0.6932(5)$ & $-0.0813(4)$ & $0.0385(11)$ \\
\hline $\mathrm{N} 2$ & $0.5196(2)$ & $0.2939(5)$ & $0.3175(5)$ & $0.0264(11)$ \\
\hline $\mathrm{N} 4$ & $0.3258(2)$ & $0.4838(5)$ & $0.5107(4)$ & $0.0271(11)$ \\
\hline N7 & $0.0466(2)$ & $0.6547(5)$ & $0.0287(4)$ & $0.0309(12)$ \\
\hline N15 & $0.4359(2)$ & $0.4704(5)$ & $-0.1171(4)$ & $0.0264(11)$ \\
\hline N16 & $0.2283(2)$ & $0.6451(5)$ & $-0.2394(4)$ & $0.0317(12)$ \\
\hline $\mathrm{C} 1$ & $0.4162(2)$ & $0.4011(6)$ & $0.1842(5)$ & $0.0216(12)$ \\
\hline H1 & 0.4389 & 0.3905 & 0.1133 & $0.026^{*}$ \\
\hline $\mathrm{C} 2$ & $0.4497(2)$ & $0.3643(5)$ & $0.3044(5)$ & $0.0198(12)$ \\
\hline $\mathrm{C} 3$ & $0.4208(3)$ & $0.3903(6)$ & $0.4109(5)$ & $0.0246(12)$ \\
\hline $\mathrm{H} 3$ & 0.4471 & 0.3747 & 0.4920 & $0.029 *$ \\
\hline $\mathrm{C} 4$ & $0.3523(2)$ & $0.4397(5)$ & $0.3960(5)$ & $0.0202(12)$ \\
\hline $\mathrm{C} 5$ & $0.1783(3)$ & $0.4889(6)$ & $0.2876(5)$ & $0.0245(13)$ \\
\hline C6 & $0.1152(2)$ & $0.5433(6)$ & $0.2194(5)$ & $0.0272(13)$ \\
\hline H6 & 0.0741 & 0.5440 & 0.2561 & $0.033^{*}$ \\
\hline $\mathrm{C} 7$ & $0.1136(2)$ & $0.5954(6)$ & $0.0985(5)$ & $0.0225(12)$ \\
\hline $\mathrm{C} 8$ & $0.1697(2)$ & $0.5900(6)$ & $0.0349(5)$ & $0.0252(13)$ \\
\hline $\mathrm{H} 8$ & 0.1657 & 0.6200 & -0.0506 & $0.030^{*}$ \\
\hline C9 & $0.3006(2)$ & $0.5089(6)$ & $0.0615(5)$ & $0.0206(12)$ \\
\hline $\mathrm{C} 10$ & $0.3476(2)$ & $0.4543(5)$ & $0.1728(4)$ & $0.0190(12)$ \\
\hline C11 & $0.3132(2)$ & $0.4608(5)$ & 0.2780 & $0.0191(12)$ \\
\hline $\mathrm{C} 12$ & $0.2382(3)$ & $0.4980(6)$ & $0.2302(5)$ & $0.0237(12)$ \\
\hline $\mathrm{C} 13$ & $0.2325(2)$ & $0.5381(6)$ & $0.1038(5)$ & $0.0204(12)$ \\
\hline C14 & $0.3158(2)$ & $0.5339(6)$ & $-0.0545(5)$ & $0.0222(12)$ \\
\hline $\mathrm{C} 15$ & $0.3831(3)$ & $0.4987(6)$ & $-0.0877(5)$ & $0.0224(12)$ \\
\hline $\mathrm{C} 16$ & $0.2665(3)$ & $0.5949(6)$ & $-0.1573(5)$ & $0.0235(12)$ \\
\hline $\mathrm{C} 17$ & $0.1782(2)$ & $0.4036(6)$ & $0.4085(5)$ & $0.0261(13)$ \\
\hline $\mathrm{C} 18$ & $0.1288(3)$ & $0.3746(6)$ & $0.5921(4)$ & $0.0294(14)$ \\
\hline H181 & 0.1753 & 0.3702 & 0.6450 & $0.038^{*}$ \\
\hline H182 & 0.1123 & 0.2671 & 0.5734 & $0.038^{*}$ \\
\hline
\end{tabular}




\begin{tabular}{lllll} 
C19 & $0.0786(3)$ & $0.4606(7)$ & $0.6594(5)$ & $0.0380(15)$ \\
H191 & 0.0952 & 0.5670 & 0.6766 & $0.049(10)^{*}$ \\
H192 & 0.0753 & 0.4081 & 0.7385 & $0.049(10)^{*}$ \\
H193 & 0.0327 & 0.4629 & 0.6066 & $0.049(10)^{*}$ \\
\hline
\end{tabular}

Atomic displacement parameters $\left(\AA^{2}\right)$

\begin{tabular}{|c|c|c|c|c|c|c|}
\hline & $U^{11}$ & $U^{22}$ & $U^{33}$ & $U^{12}$ & $U^{13}$ & $U^{23}$ \\
\hline $\mathrm{O} 1$ & $0.025(2)$ & $0.034(2)$ & $0.036(2)$ & $0.0071(17)$ & $0.0116(17)$ & 0.0019 (19) \\
\hline $\mathrm{O} 2$ & $0.022(2)$ & 0.039 (3) & $0.031(2)$ & $0.0037(18)$ & $-0.0046(18)$ & $0.0040(18)$ \\
\hline $\mathrm{O} 3$ & $0.034(2)$ & $0.044(3)$ & $0.019(2)$ & 0.0073 (19) & $-0.0010(16)$ & $0.0118(19)$ \\
\hline $\mathrm{O} 4$ & $0.023(2)$ & $0.038(2)$ & $0.028(2)$ & 0.0037 (19) & $0.0053(16)$ & $-0.0030(18)$ \\
\hline $\mathrm{O} 5$ & $0.030(2)$ & $0.028(2)$ & $0.040(2)$ & $0.0083(19)$ & $0.0079(17)$ & $0.0064(18)$ \\
\hline O6 & $0.022(2)$ & $0.038(2)$ & 0.031 (2) & $0.0013(17)$ & $0.0063(17)$ & $0.0005(18)$ \\
\hline $\mathrm{O} 7$ & $0.019(2)$ & 0.058 (3) & $0.046(3)$ & $0.0080(19)$ & $0.0083(19)$ & 0.014 (2) \\
\hline O8 & $0.023(2)$ & $0.062(3)$ & $0.028(2)$ & $0.0122(19)$ & $-0.0031(17)$ & $0.006(2)$ \\
\hline N2 & $0.022(2)$ & $0.021(3)$ & $0.036(3)$ & $-0.001(2)$ & $0.004(2)$ & $0.006(2)$ \\
\hline N4 & $0.022(3)$ & $0.030(3)$ & $0.029(3)$ & $0.004(2)$ & $0.001(2)$ & $0.004(2)$ \\
\hline N7 & $0.020(3)$ & 0.039 (3) & 0.033 & $0.005(2)$ & $0.001(2)$ & $0.005(2)$ \\
\hline N15 & $0.021(3)$ & $0.033(3)$ & 0.025 & $-0.001(2)$ & $0.005(2)$ & $-0.001(2)$ \\
\hline N16 & $0.025(3)$ & $0.040(3)$ & $0.030(3)$ & $0.003(2)$ & $0.004(2)$ & $0.003(2)$ \\
\hline $\mathrm{C} 1$ & 0.019 (3) & 0.015 & $0.030(3)$ & $0.000(2)$ & $0.001(2)$ & $0.000(2)$ \\
\hline $\mathrm{C} 2$ & 0.019 (3) & $0.013(3)$ & 0.028 & $0.003(2)$ & $0.005(2)$ & $0.003(2)$ \\
\hline $\mathrm{C} 3$ & $0.025(3)$ & $0.022(3)$ & $0.024(3)$ & $-0.002(2)$ & $-0.002(2)$ & $0.003(2)$ \\
\hline $\mathrm{C} 4$ & $0.021(3)$ & 0.015 (3) & $0.023(3)$ & $0.002(2)$ & $0.001(2)$ & $-0.001(2)$ \\
\hline $\mathrm{C} 5$ & 0.019 (3) & 0.028 & $0.028(3)$ & $0.003(2)$ & 0.008 (2) & $0.001(2)$ \\
\hline C6 & $0.015(3)$ & $0.036(4)$ & $0.031(3)$ & $0.000(2)$ & $0.005(2)$ & $-0.003(3)$ \\
\hline $\mathrm{C} 7$ & 0.015 (3) & 0.029 (3) & $0.023(3)$ & $0.003(2)$ & $-0.001(2)$ & $0.000(2)$ \\
\hline $\mathrm{C} 8$ & $0.021(3)$ & $0.033(3)$ & $0.020(3)$ & $-0.004(2)$ & $-0.001(2)$ & $-0.001(2)$ \\
\hline $\mathrm{C} 9$ & $0.013(3)$ & 0.019 (3) & $0.030(3)$ & $-0.002(2)$ & $0.003(2)$ & $-0.005(2)$ \\
\hline $\mathrm{C} 10$ & $0.014(3)$ & 0.019 (3) & $0.023(3)$ & $-0.003(2)$ & $0.000(2)$ & $-0.002(2)$ \\
\hline C11 & $0.018(3)$ & $0.016(3)$ & $0.024(3)$ & $0.004(2)$ & $0.006(2)$ & $-0.001(2)$ \\
\hline $\mathrm{C} 12$ & $0.022(3)$ & $0.023(3)$ & $0.025(3)$ & $0.005(2)$ & $0.001(2)$ & $-0.002(2)$ \\
\hline $\mathrm{C} 13$ & $0.020(3)$ & 0.019 (3) & $0.022(3)$ & $-0.002(2)$ & $0.001(2)$ & $-0.001(2)$ \\
\hline $\mathrm{C} 14$ & $0.021(3)$ & $0.022(3)$ & $0.024(3)$ & $-0.003(2)$ & $0.003(2)$ & $0.000(2)$ \\
\hline $\mathrm{C} 15$ & $0.023(3)$ & $0.025(3)$ & 0.018 & $-0.002(2)$ & $-0.002(2)$ & $0.003(2)$ \\
\hline C16 & $0.018(3)$ & 0.029 (3) & $0.023(3)$ & $-0.001(2)$ & $0.001(2)$ & -0.005 (3) \\
\hline $\mathrm{C} 17$ & $0.012(3)$ & $0.032(4)$ & $0.034(3)$ & -0.003 & $0.004(2)$ & $-0.002(3)$ \\
\hline $\mathrm{C} 18$ & $0.028(3)$ & $0.040(4)$ & $0.020(3)$ & $-0.004(3)$ & $0.001(2)$ & 0.007 (3) \\
\hline C19 & $0.036(3)$ & $0.052(4)$ & $0.027(3)$ & $-0.007(3)$ & $0.010(3)$ & $0.002(3)$ \\
\hline
\end{tabular}

Geometric parameters $\left(\AA,{ }^{\circ}\right)$

\begin{tabular}{llll}
\hline $\mathrm{O} 1-\mathrm{N} 2$ & $1.234(5)$ & $\mathrm{C} 5-\mathrm{C} 12$ & $1.412(7)$ \\
$\mathrm{O} 2-\mathrm{N} 2$ & $1.228(5)$ & $\mathrm{C} 5-\mathrm{C} 17$ & $1.500(7)$ \\
$\mathrm{O} 3-\mathrm{N} 4$ & $1.231(5)$ & $\mathrm{C} 6-\mathrm{C} 7$ & $1.378(7)$ \\
$\mathrm{O} 4-\mathrm{N} 4$ & $1.235(5)$ & $\mathrm{C} 6-\mathrm{H} 6$ & 0.9500 \\
$\mathrm{O} 5-\mathrm{C} 17$ & $1.217(6)$ & $\mathrm{C} 7-\mathrm{C} 8$ & $1.384(6)$
\end{tabular}




\begin{tabular}{|c|c|c|c|}
\hline $\mathrm{O} 6-\mathrm{C} 17$ & $1.317(6)$ & $\mathrm{C} 8-\mathrm{C} 13$ & $1.398(7)$ \\
\hline $\mathrm{O} 6-\mathrm{C} 18$ & $1.474(6)$ & $\mathrm{C} 8-\mathrm{H} 8$ & 0.9500 \\
\hline $\mathrm{O} 7-\mathrm{N} 7$ & $1.228(5)$ & $\mathrm{C} 9-\mathrm{C} 14$ & $1.352(7)$ \\
\hline $\mathrm{O} 8-\mathrm{N} 7$ & $1.230(5)$ & $\mathrm{C} 9-\mathrm{C} 10$ & $1.465(7)$ \\
\hline $\mathrm{N} 2-\mathrm{C} 2$ & $1.476(6)$ & $\mathrm{C} 9-\mathrm{C} 13$ & $1.495(6)$ \\
\hline $\mathrm{N} 4-\mathrm{C} 4$ & $1.469(6)$ & $\mathrm{C} 10-\mathrm{C} 11$ & $1.412(6)$ \\
\hline $\mathrm{N} 7-\mathrm{C} 7$ & $1.487(6)$ & $\mathrm{C} 11-\mathrm{C} 12$ & $1.502(7)$ \\
\hline $\mathrm{N} 15-\mathrm{C} 15$ & $1.152(6)$ & $\mathrm{C} 12-\mathrm{C} 13$ & $1.397(7)$ \\
\hline $\mathrm{N} 16-\mathrm{C} 16$ & $1.147(6)$ & $\mathrm{C} 14-\mathrm{C} 16$ & $1.444(7)$ \\
\hline $\mathrm{C} 1-\mathrm{C} 2$ & $1.393(6)$ & $\mathrm{C} 14-\mathrm{C} 15$ & $1.446(7)$ \\
\hline $\mathrm{C} 1-\mathrm{C} 10$ & $1.401(6)$ & $\mathrm{C} 18-\mathrm{C} 19$ & $1.505(7)$ \\
\hline $\mathrm{C} 1-\mathrm{H} 1$ & 0.9501 & C18-H181 & 0.9900 \\
\hline $\mathrm{C} 2-\mathrm{C} 3$ & $1.381(6)$ & C18-H182 & 0.9900 \\
\hline $\mathrm{C} 3-\mathrm{C} 4$ & $1.385(6)$ & C19-H191 & 0.9800 \\
\hline $\mathrm{C} 3-\mathrm{H} 3$ & 0.9500 & C19-H192 & 0.9800 \\
\hline $\mathrm{C} 4-\mathrm{C} 11$ & $1.388(6)$ & C19-H193 & 0.9799 \\
\hline $\mathrm{C} 5-\mathrm{C} 6$ & $1.407(7)$ & & \\
\hline $\mathrm{C} 17-\mathrm{O} 6-\mathrm{C} 18$ & $115.1(4)$ & $\mathrm{C} 14-\mathrm{C} 9-\mathrm{C} 13$ & $126.6(4)$ \\
\hline $\mathrm{O} 2-\mathrm{N} 2-\mathrm{O} 1$ & $124.6(4)$ & $\mathrm{C} 10-\mathrm{C} 9-\mathrm{C} 13$ & $105.6(4)$ \\
\hline $\mathrm{O} 2-\mathrm{N} 2-\mathrm{C} 2$ & $118.1(4)$ & $\mathrm{C} 1-\mathrm{C} 10-\mathrm{C} 11$ & $120.9(4)$ \\
\hline $\mathrm{O} 1-\mathrm{N} 2-\mathrm{C} 2$ & $117.3(4)$ & $\mathrm{C} 1-\mathrm{C} 10-\mathrm{C} 9$ & $129.5(4)$ \\
\hline $\mathrm{O} 3-\mathrm{N} 4-\mathrm{O} 4$ & $124.7(4)$ & $\mathrm{C} 11-\mathrm{C} 10-\mathrm{C} 9$ & $109.6(4)$ \\
\hline $\mathrm{O} 3-\mathrm{N} 4-\mathrm{C} 4$ & $118.1(4)$ & $\mathrm{C} 4-\mathrm{C} 11-\mathrm{C} 10$ & $118.3(4)$ \\
\hline $\mathrm{O} 4-\mathrm{N} 4-\mathrm{C} 4$ & $117.1(4)$ & $\mathrm{C} 4-\mathrm{C} 11-\mathrm{C} 12$ & $134.5(4)$ \\
\hline $\mathrm{O} 7-\mathrm{N} 7-\mathrm{O} 8$ & $124.5(4)$ & $\mathrm{C} 10-\mathrm{C} 11-\mathrm{C} 12$ & $107.1(4)$ \\
\hline $\mathrm{O} 7-\mathrm{N} 7-\mathrm{C} 7$ & $117.6(4)$ & $\mathrm{C} 13-\mathrm{C} 12-\mathrm{C} 5$ & $120.3(4)$ \\
\hline $\mathrm{O} 8-\mathrm{N} 7-\mathrm{C} 7$ & $117.9(4)$ & $\mathrm{C} 13-\mathrm{C} 12-\mathrm{C} 11$ & $107.9(4)$ \\
\hline $\mathrm{C} 2-\mathrm{C} 1-\mathrm{C} 10$ & $116.9(4)$ & $\mathrm{C} 5-\mathrm{C} 12-\mathrm{C} 11$ & $131.6(5)$ \\
\hline $\mathrm{C} 2-\mathrm{C} 1-\mathrm{H} 1$ & 121.5 & $\mathrm{C} 12-\mathrm{C} 13-\mathrm{C} 8$ & $121.6(4)$ \\
\hline $\mathrm{C} 10-\mathrm{C} 1-\mathrm{H} 1$ & 121.7 & $\mathrm{C} 12-\mathrm{C} 13-\mathrm{C} 9$ & $108.9(4)$ \\
\hline $\mathrm{C} 3-\mathrm{C} 2-\mathrm{C} 1$ & $123.3(4)$ & $\mathrm{C} 8-\mathrm{C} 13-\mathrm{C} 9$ & $129.4(5)$ \\
\hline $\mathrm{C} 3-\mathrm{C} 2-\mathrm{N} 2$ & $118.8(4)$ & $\mathrm{C} 9-\mathrm{C} 14-\mathrm{C} 16$ & $123.6(4)$ \\
\hline $\mathrm{C} 1-\mathrm{C} 2-\mathrm{N} 2$ & $117.9(4)$ & $\mathrm{C} 9-\mathrm{C} 14-\mathrm{C} 15$ & $122.7(4)$ \\
\hline $\mathrm{C} 2-\mathrm{C} 3-\mathrm{C} 4$ & $118.0(5)$ & $\mathrm{C} 16-\mathrm{C} 14-\mathrm{C} 15$ & $113.7(4)$ \\
\hline $\mathrm{C} 2-\mathrm{C} 3-\mathrm{H} 3$ & 121.0 & $\mathrm{~N} 15-\mathrm{C} 15-\mathrm{C} 14$ & $178.3(5)$ \\
\hline $\mathrm{C} 4-\mathrm{C} 3-\mathrm{H} 3$ & 121.0 & $\mathrm{~N} 16-\mathrm{C} 16-\mathrm{C} 14$ & $178.7(6)$ \\
\hline $\mathrm{C} 3-\mathrm{C} 4-\mathrm{C} 11$ & $121.4(4)$ & $\mathrm{O} 5-\mathrm{C} 17-\mathrm{O} 6$ & $125.0(5)$ \\
\hline $\mathrm{C} 3-\mathrm{C} 4-\mathrm{N} 4$ & $116.5(4)$ & $\mathrm{O} 5-\mathrm{C} 17-\mathrm{C} 5$ & $121.7(5)$ \\
\hline $\mathrm{C} 11-\mathrm{C} 4-\mathrm{N} 4$ & $121.8(4)$ & $\mathrm{O} 6-\mathrm{C} 17-\mathrm{C} 5$ & $113.2(5)$ \\
\hline $\mathrm{C} 6-\mathrm{C} 5-\mathrm{C} 12$ & $117.7(5)$ & $\mathrm{O} 6-\mathrm{C} 18-\mathrm{C} 19$ & $107.6(4)$ \\
\hline $\mathrm{C} 6-\mathrm{C} 5-\mathrm{C} 17$ & $119.7(4)$ & $\mathrm{O} 6-\mathrm{C} 18-\mathrm{H} 181$ & 110.2 \\
\hline $\mathrm{C} 12-\mathrm{C} 5-\mathrm{C} 17$ & $122.0(4)$ & $\mathrm{C} 19-\mathrm{C} 18-\mathrm{H} 181$ & 110.1 \\
\hline $\mathrm{C} 7-\mathrm{C} 6-\mathrm{C} 5$ & $119.2(5)$ & $\mathrm{O} 6-\mathrm{C} 18-\mathrm{H} 182$ & 110.3 \\
\hline $\mathrm{C} 7-\mathrm{C} 6-\mathrm{H} 6$ & 120.3 & $\mathrm{C} 19-\mathrm{C} 18-\mathrm{H} 182$ & 110.2 \\
\hline $\mathrm{C} 5-\mathrm{C} 6-\mathrm{H} 6$ & 120.4 & $\mathrm{H} 181-\mathrm{C} 18-\mathrm{H} 182$ & 108.5 \\
\hline $\mathrm{C} 6-\mathrm{C} 7-\mathrm{C} 8$ & $124.3(5)$ & C18-C19-H191 & 109.5 \\
\hline $\mathrm{C} 6-\mathrm{C} 7-\mathrm{N} 7$ & $118.4(4)$ & $\mathrm{C} 18-\mathrm{C} 19-\mathrm{H} 192$ & 109.6 \\
\hline
\end{tabular}




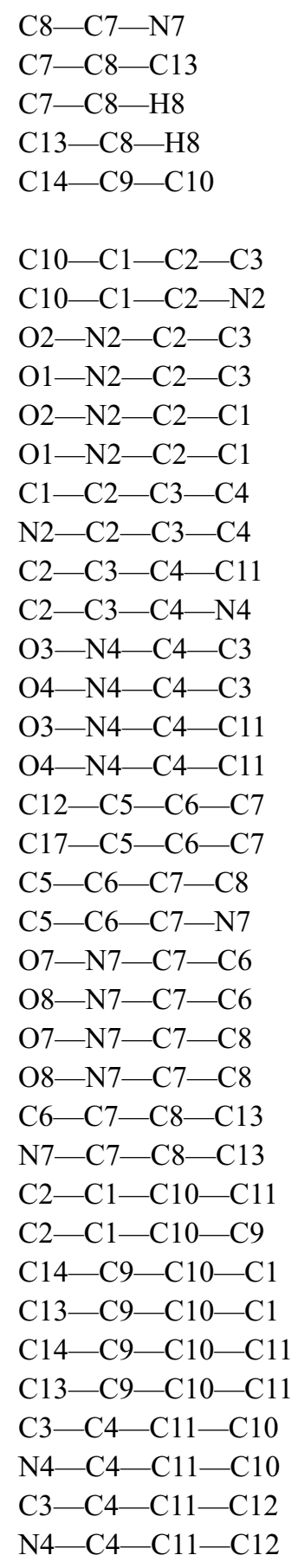

$117.2(5)$

$116.0(5)$

122.0

122.0

127.7 (4)

$-5.7(7)$

$174.2(4)$

$-19.5(6)$

$160.0(4)$

$160.6(4)$

$-19.9(6)$

$7.2(7)$

$-172.7(4)$

$0.8(7)$

$-173.0(4)$

$-33.3(6)$

$143.6(4)$

$152.9(5)$

$-30.2(6)$

$3.2(8)$

$-168.0(5)$

$4.0(8)$

$-179.1(4)$

4.4 (7)

$-176.2(5)$

$-178.4(5)$

$1.0(7)$

$-4.8(8)$

$178.2(4)$

$-3.7(7)$

$176.5(5)$

$-6.9(9)$

$175.5(5)$

$173.3(5)$

$-4.3(5)$

$-9.7(7)$

$163.8(4)$

$173.5(5)$

$-13.0(8)$

$$
\begin{aligned}
& \text { H191-C19-H192 } \\
& \text { C18-C19-H193 } \\
& \text { H191-C19-H193 } \\
& \text { H192-C19-H193 }
\end{aligned}
$$

$\mathrm{C} 1-\mathrm{C} 10-\mathrm{C} 11-\mathrm{C} 4$

C9- $\mathrm{C} 10-\mathrm{C} 11-\mathrm{C} 4$

$\mathrm{C} 1-\mathrm{C} 10-\mathrm{C} 11-\mathrm{C} 12$

$\mathrm{C} 9-\mathrm{C} 10-\mathrm{C} 11-\mathrm{C} 12$

$\mathrm{C} 6-\mathrm{C} 5-\mathrm{C} 12-\mathrm{C} 13$

$\mathrm{C} 17-\mathrm{C} 5-\mathrm{C} 12-\mathrm{C} 13$

$\mathrm{C} 6-\mathrm{C} 5-\mathrm{C} 12-\mathrm{C} 11$

$\mathrm{C} 17-\mathrm{C} 5-\mathrm{C} 12-\mathrm{C} 11$

$\mathrm{C} 4-\mathrm{C} 11-\mathrm{C} 12-\mathrm{C} 13$

$\mathrm{C} 10-\mathrm{C} 11-\mathrm{C} 12-\mathrm{C} 13$

$\mathrm{C} 4-\mathrm{C} 11-\mathrm{C} 12-\mathrm{C} 5$

$\mathrm{C} 10-\mathrm{C} 11-\mathrm{C} 12-\mathrm{C} 5$

$\mathrm{C} 5-\mathrm{C} 12-\mathrm{C} 13-\mathrm{C} 8$

$\mathrm{C} 11-\mathrm{C} 12-\mathrm{C} 13-\mathrm{C} 8$

$\mathrm{C} 5-\mathrm{C} 12-\mathrm{C} 13-\mathrm{C} 9$

$\mathrm{C} 11-\mathrm{C} 12-\mathrm{C} 13-\mathrm{C} 9$

$\mathrm{C} 7-\mathrm{C} 8-\mathrm{C} 13-\mathrm{C} 12$

$\mathrm{C} 7-\mathrm{C} 8-\mathrm{C} 13-\mathrm{C} 9$

$\mathrm{C} 14-\mathrm{C} 9-\mathrm{C} 13-\mathrm{C} 12$

$\mathrm{C} 10-\mathrm{C} 9-\mathrm{C} 13-\mathrm{C} 12$

$\mathrm{C} 14-\mathrm{C} 9-\mathrm{C} 13-\mathrm{C} 8$

$\mathrm{C} 10-\mathrm{C} 9-\mathrm{C} 13-\mathrm{C} 8$

$\mathrm{C} 10-\mathrm{C} 9-\mathrm{C} 14-\mathrm{C} 16$

$\mathrm{C} 13-\mathrm{C} 9-\mathrm{C} 14-\mathrm{C} 16$

$\mathrm{C} 10-\mathrm{C} 9-\mathrm{C} 14-\mathrm{C} 15$

$\mathrm{C} 13-\mathrm{C} 9-\mathrm{C} 14-\mathrm{C} 15$

$\mathrm{C} 18-\mathrm{O} 6-\mathrm{C} 17-\mathrm{O} 5$

$\mathrm{C} 18-\mathrm{O} 6-\mathrm{C} 17-\mathrm{C} 5$

$\mathrm{C} 6-\mathrm{C} 5-\mathrm{C} 17-\mathrm{O} 5$

$\mathrm{C} 12-\mathrm{C} 5-\mathrm{C} 17-\mathrm{O} 5$

$\mathrm{C} 6-\mathrm{C} 5-\mathrm{C} 17-\mathrm{O} 6$

$\mathrm{C} 12-\mathrm{C} 5-\mathrm{C} 17-\mathrm{O} 6$

$\mathrm{C} 17-\mathrm{O} 6-\mathrm{C} 18-\mathrm{C} 19$
109.5

109.4

109.5

109.5

$11.1(7)$

$-169.1(4)$

$-171.2(4)$

$8.6(5)$

$-9.2(7)$

$161.8(5)$

$175.6(5)$

$-13.4(9)$

$167.2(5)$

$-9.9(5)$

$-17.1(10)$

$165.8(5)$

8.5 (8)

-175.2 (4)

-169.0 (4)

$7.2(5)$

$-1.5(7)$

$175.5(5)$

$-179.7(5)$

$-2.0(5)$

$3.0(8)$

-179.4 (5)

$-177.0(5)$

$0.1(8)$

$4.3(8)$

$-178.5(5)$

0.3 (7)

$177.6(4)$

$140.5(5)$

$-30.4(8)$

$-36.9(7)$

$152.2(5)$

$175.8(4)$ 